\title{
Categorical Pullbacks
}

\author{
Marco Riccardi \\ Via del Pero 102 \\ 54038 Montignoso \\ Italy
}

\begin{abstract}
Summary. The main purpose of this article is to introduce the categorical concept of pullback in Mizar. In the first part of this article we redefine homsets, monomorphisms, epimorpshisms and isomorphisms [7] within a free-object category [1] and it is shown there that ordinal numbers can be considered as categories. Then the pullback is introduced in terms of its universal property and the Pullback Lemma is formalized [15. In the last part of the article we formalize the pullback of functors [14] and it is also shown that it is not possible to write an equivalent definition in the context of the previous Mizar formalization of category theory [8].
\end{abstract}

MSC: 18A30 03B35

Keywords: category pullback; pullback lemma

MML identifier: CAT_7, version: 8.1.03 5.29.1227

The notation and terminology used in this paper have been introduced in the following articles: [2], [8, [17, [18, [6], [13], 9], [10], [3], 11], 20], 21], [16], [19], 4], [5], and [12].

\section{PReliminaries}

One can verify that every set which is ordinal is also non pair.

Let $\mathscr{C}$ be an empty category structure. Let us note that Mor $\mathscr{C}$ is empty.

Let $\mathscr{C}$ be a non empty category structure. Note that Mor $\mathscr{C}$ is non empty.

Let $\mathscr{C}$ be an empty category structure with identities. Let us note that $\mathrm{Ob} \mathscr{C}$ is empty.

Let $\mathscr{C}$ be a non empty category structure with identities. Observe that $\mathrm{Ob} \mathscr{C}$ is non empty. 
Let $\mathscr{C}$ be category structure with identities and $a$ be an object of $\mathscr{C}$. One can check that id- $a$ is identity.

Now we state the propositions:

(1) Let us consider a category structure $\mathscr{C}$, and a morphism $f$ of $\mathscr{C}$. Suppose $\mathscr{C}$ is not empty. Then $f \in$ the carrier of $\mathscr{C}$.

(2) Let us consider category structure $\mathscr{C}$ with identities, and an object $a$ of $\mathscr{C}$. Suppose $\mathscr{C}$ is not empty. Then $a \in$ the carrier of $\mathscr{C}$.

(3) Let us consider a composable category structure $\mathscr{C}$, and morphisms $f_{1}$, $f_{2}, f_{3}$ of $\mathscr{C}$. Suppose $f_{1} \triangleright f_{2}$ and $f_{2} \triangleright f_{3}$ and $f_{2}$ is identity. Then $f_{1} \triangleright f_{3}$.

(4) Let us consider a composable category structure $\mathscr{C}$ with identities, and morphisms $f_{1}, f_{2}$ of $\mathscr{C}$. Suppose $f_{1} \triangleright f_{2}$. Then

(i) $\operatorname{dom}\left(f_{1} \circ f_{2}\right)=\operatorname{dom} f_{2}$, and

(ii) $\operatorname{cod}\left(f_{1} \circ f_{2}\right)=\operatorname{cod} f_{1}$.

(5) Let us consider a non empty, composable category structure $\mathscr{C}$ with identities, and morphisms $f_{1}, f_{2}$ of $\mathscr{C}$. Then $f_{1} \triangleright f_{2}$ if and only if $\operatorname{dom} f_{1}=$ $\operatorname{cod} f_{2}$.

(6) Let us consider a composable category structure $\mathscr{C}$ with identities, and a morphism $f$ of $\mathscr{C}$. If $f$ is identity, then $\operatorname{dom} f=f$ and $\operatorname{cod} f=f$.

(7) Let us consider a composable category structure $\mathscr{C}$ with identities, and morphisms $f_{1}, f_{2}$ of $\mathscr{C}$. Suppose $f_{1} \triangleright f_{2}$ and $f_{1}$ is identity and $f_{2}$ is identity. Then $f_{1}=f_{2}$.

Let us consider a non empty, composable category structure $\mathscr{C}$ with identities and morphisms $f_{1}, f_{2}$ of $\mathscr{C}$. Now we state the propositions:

(8) If $\operatorname{dom} f_{1}=f_{2}$, then $f_{1} \triangleright f_{2}$ and $f_{1} \circ f_{2}=f_{1}$.

(9) If $f_{1}=\operatorname{cod} f_{2}$, then $f_{1} \triangleright f_{2}$ and $f_{1} \circ f_{2}=f_{2}$.

Now we state the propositions:

(10) Let us consider categories $\mathscr{C}_{1}, \mathscr{C}_{2}, \mathscr{C}_{3}, \mathscr{C}_{4}$, a functor $\mathcal{F}$ from $\mathscr{C}_{1}$ to $\mathscr{C}_{2}$, a functor $\mathcal{G}$ from $\mathscr{C}_{2}$ to $\mathscr{C}_{3}$, and a functor $\mathcal{H}$ from $\mathscr{C}_{3}$ to $\mathscr{C}_{4}$. Suppose $\mathcal{F}$ is covariant and $\mathcal{G}$ is covariant and $\mathcal{H}$ is covariant. Then $\mathcal{H} \circ(\mathcal{G} \circ \mathcal{F})=$ $(\mathcal{H} \circ \mathcal{G}) \circ \mathcal{F}$.

(11) Let us consider categories $\mathscr{C}, \mathscr{D}$, and a functor $\mathcal{F}$ from $\mathscr{C}$ to $\mathscr{D}$. Suppose $\mathcal{F}$ is covariant. Then

(i) $\mathcal{F} \circ \operatorname{id}_{\mathscr{C}}=\mathcal{F}$, and

(ii) $\operatorname{id}_{\mathscr{D}} \circ \mathcal{F}=\mathcal{F}$.

(12) Let us consider composable category structures $\mathscr{C}, \mathscr{D}$ with identities. Then $\mathscr{C} \cong \mathscr{D}$ if and only if there exists a functor $\mathcal{F}$ from $\mathscr{C}$ to $\mathscr{D}$ such that $\mathcal{F}$ is covariant and bijective. The theorem is a consequence of (5). 
(13) Let us consider empty category structures $\mathscr{C}, \mathscr{D}$ with identities. Then $\mathscr{C} \cong \mathscr{D}$.

Let us consider category structures $\mathscr{C}, \mathscr{D}$ with identities. Now we state the propositions:

(14) Suppose $\mathscr{C} \cong \mathscr{D}$. Then

(i) $\overline{\overline{\operatorname{Mor} \mathscr{C}}}=\overline{\overline{\text { Mor } \mathscr{D}}}$, and

(ii) $\overline{\overline{\mathrm{Ob} \mathscr{C}}}=\overline{\overline{\mathrm{Ob} \mathscr{D}}}$.

(15) If $\mathscr{C} \cong \mathscr{D}$ and $\mathscr{C}$ is empty, then $\mathscr{D}$ is empty. The theorem is a consequence of $(14)$.

\section{HOM-SETS}

Let $\mathscr{C}$ be a category structure and $a, b$ be objects of $\mathscr{C}$. The functor hom $(a, b)$ yielding a subset of Mor $\mathscr{C}$ is defined by the term

(Def. 1) $\left\{f\right.$, where $f$ is a morphism of $\mathscr{C}$ : there exist morphisms $f_{1}, f_{2}$ of $\mathscr{C}$ such that $a=f_{1}$ and $b=f_{2}$ and $f \triangleright f_{1}$ and $\left.f_{2} \triangleright f\right\}$.

Let $\mathscr{C}$ be a non empty, composable category structure with identities. Observe that the functor $\operatorname{hom}(a, b)$ yields a subset of Mor $\mathscr{C}$ and is defined by the term

(Def. 2) $\{f$, where $f$ is a morphism of $\mathscr{C}: \operatorname{dom} f=a$ and $\operatorname{cod} f=b\}$.

Let $\mathscr{C}$ be a category structure. Assume $\operatorname{hom}(a, b) \neq \emptyset$.

A morphism from $a$ to $b$ is a morphism of $\mathscr{C}$ and is defined by

(Def. 3) it $\in \operatorname{hom}(a, b)$.

Let $\mathscr{C}$ be category structure with identities and $a$ be an object of $\mathscr{C}$. Assume $\operatorname{hom}(a, a) \neq \emptyset$. Observe that the functor id- $a$ yields a morphism from $a$ to $a$. Let $\mathscr{C}$ be a non empty category structure with identities. Note that $\operatorname{hom}(a, a)$ is non empty.

Let $\mathscr{C}$ be a composable category structure with identities, $a, b, c$ be objects of $\mathscr{C}, f$ be a morphism from $a$ to $b$, and $g$ be a morphism from $b$ to $c$. Assume $\operatorname{hom}(a, b) \neq \emptyset$ and $\operatorname{hom}(b, c) \neq \emptyset$. The functor $g \cdot f$ yielding a morphism from $a$ to $c$ is defined by the term

(Def. 4) $g \circ f$.

Now we state the propositions:

(16) Let us consider a category structure $\mathscr{C}$, objects $a, b$ of $\mathscr{C}$, and a morphism $f$ from $a$ to $b$. Suppose $\operatorname{hom}(a, b) \neq \emptyset$. Then there exist morphisms $f_{1}, f_{2}$ of $\mathscr{C}$ such that

(i) $a=f_{1}$, and 
(ii) $b=f_{2}$, and

(iii) $f \triangleright f_{1}$, and

(iv) $f_{2} \triangleright f$.

(17) Let us consider a composable category structure $\mathscr{C}$ with identities, objects $a, b, c$ of $\mathscr{C}$, a morphism $f_{1}$ from $a$ to $b$, and a morphism $f_{2}$ from $b$ to $c$. Suppose $\operatorname{hom}(a, b) \neq \emptyset$ and $\operatorname{hom}(b, c) \neq \emptyset$. Then $f_{2} \triangleright f_{1}$. The theorem is a consequence of (16) and (3).

(18) Let us consider a composable category structure $\mathscr{C}$ with identities, objects $a, b$ of $\mathscr{C}$, and a morphism $f$ from $a$ to $b$. Suppose $\operatorname{hom}(a, b) \neq \emptyset$. Then

(i) $f \cdot \mathrm{id}-a=f$, and

(ii) id- $b \cdot f=f$.

The theorem is a consequence of (17).

(19) Let us consider a non empty, composable category structure $\mathscr{C}$ with identities, and a morphism $f$ of $\mathscr{C}$. Then $f \in \operatorname{hom}(\operatorname{dom} f, \operatorname{cod} f)$.

(20) Let us consider a non empty, composable category structure $\mathscr{C}$ with identities, objects $a, b$ of $\mathscr{C}$, and a morphism $f$ of $\mathscr{C}$. Then $f \in \operatorname{hom}(a, b)$ if and only if $\operatorname{dom} f=a$ and $\operatorname{cod} f=b$.

(21) Let us consider a non empty, composable category structure $\mathscr{C}$ with identities, and an object $a$ of $\mathscr{C}$. Then $a \in \operatorname{hom}(a, a)$. The theorem is a consequence of $(6)$.

(22) Let us consider a composable category structure $\mathscr{C}$ with identities, and objects $a, b, c$ of $\mathscr{C}$. Suppose $\operatorname{hom}(a, b) \neq \emptyset$ and $\operatorname{hom}(b, c) \neq \emptyset$. Then $\operatorname{hom}(a, c) \neq \emptyset$. The theorem is a consequence of (16) and (3).

(23) Let us consider a category $\mathscr{C}$, objects $a, b, c, d$ of $\mathscr{C}$, a morphism $f_{1}$ from $a$ to $b$, a morphism $f_{2}$ from $b$ to $c$, and a morphism $f_{3}$ from $c$ to $d$. Suppose $\operatorname{hom}(a, b) \neq \emptyset$ and $\operatorname{hom}(b, c) \neq \emptyset$ and $\operatorname{hom}(c, d) \neq \emptyset$. Then $f_{3} \cdot\left(f_{2} \cdot f_{1}\right)=\left(f_{3} \cdot f_{2}\right) \cdot f_{1}$. The theorem is a consequence of $(22)$ and $(17)$.

(24) Let us consider a composable category structure $\mathscr{C}$ with identities, objects $a, b, c$ of $\mathscr{C}$, a morphism $f_{1}$ from $a$ to $b$, and a morphism $f_{2}$ from $b$ to $c$. Suppose $\operatorname{hom}(a, b) \neq \emptyset$ and $\operatorname{hom}(b, c) \neq \emptyset$. Then

(i) if $f_{1}$ is identity, then $f_{2} \cdot f_{1}=f_{2}$, and

(ii) if $f_{2}$ is identity, then $f_{2} \cdot f_{1}=f_{1}$.

PRoof: $f_{2} \triangleright f_{1}$. If $f_{1}$ is identity, then $f_{2} \cdot f_{1}=f_{2}$ by [17, $\left.(22),(23)\right]$. 


\section{Monomorphisms, Epimorphisms And IsOMORPHisms}

Let $\mathscr{C}$ be a composable category structure with identities, $a, b$ be objects of $\mathscr{C}$, and $f$ be a morphism from $a$ to $b$. We say that $f$ is monomorphic if and only if

(Def. 5) $\operatorname{hom}(a, b) \neq \emptyset$ and for every object $c$ of $\mathscr{C}$ such that hom $(c, a) \neq \emptyset$ for every morphisms $g_{1}, g_{2}$ from $c$ to $a$ such that $f \cdot g_{1}=f \cdot g_{2}$ holds $g_{1}=g_{2}$.

We say that $f$ is epimorphic if and only if

(Def. 6) $\operatorname{hom}(a, b) \neq \emptyset$ and for every object $c$ of $\mathscr{C}$ such that $\operatorname{hom}(b, c) \neq \emptyset$ for every morphisms $g_{1}, g_{2}$ from $b$ to $c$ such that $g_{1} \cdot f=g_{2} \cdot f$ holds $g_{1}=g_{2}$.

Now we state the proposition:

(25) Let us consider a composable category structure $\mathscr{C}$ with identities, objects $a, b$ of $\mathscr{C}$, and a morphism $f_{1}$ from $a$ to $b$. Suppose $\operatorname{hom}(a, b) \neq \emptyset$ and $f_{1}$ is identity. Then $f_{1}$ is monomorphic. The theorem is a consequence of (24).

Let us consider a category $\mathscr{C}$, objects $a, b, c$ of $\mathscr{C}$, a morphism $f_{1}$ from $a$ to $b$, and a morphism $f_{2}$ from $b$ to $c$. Now we state the propositions:

(26) If $f_{1}$ is monomorphic and $f_{2}$ is monomorphic, then $f_{2} \cdot f_{1}$ is monomorphic. The theorem is a consequence of (22) and (23).

(27) If $f_{2} \cdot f_{1}$ is monomorphic and $\operatorname{hom}(a, b) \neq \emptyset$ and $\operatorname{hom}(b, c) \neq \emptyset$, then $f_{1}$ is monomorphic. The theorem is a consequence of (23).

Let $\mathscr{C}$ be a composable category structure with identities, $a, b$ be objects of $\mathscr{C}$, and $f$ be a morphism from $a$ to $b$. We say that $f$ is a section if and only if

(Def. 7) $\operatorname{hom}(a, b) \neq \emptyset$ and $\operatorname{hom}(b, a) \neq \emptyset$ and there exists a morphism $g$ from $b$ to $a$ such that $g \cdot f=\mathrm{id}-a$.

We say that $f$ is a retraction if and only if

(Def. 8) $\operatorname{hom}(a, b) \neq \emptyset$ and $\operatorname{hom}(b, a) \neq \emptyset$ and there exists a morphism $g$ from $b$ to $a$ such that $f \cdot g=\mathrm{id}-b$.

Now we state the propositions:

(28) Let us consider a category $\mathscr{C}$, objects $a, b$ of $\mathscr{C}$, and a morphism $f$ from $a$ to $b$. If $f$ is a section, then $f$ is monomorphic. The theorem is a consequence of (23) and (18).

(29) Let us consider a composable category structure $\mathscr{C}$ with identities, objects $a, b$ of $\mathscr{C}$, and a morphism $f_{1}$ from $a$ to $b$. Suppose $\operatorname{hom}(a, b) \neq \emptyset$ and $f_{1}$ is identity. Then $f_{1}$ is epimorphic. The theorem is a consequence of $(24)$.

Let us consider a category $\mathscr{C}$, objects $a, b, c$ of $\mathscr{C}$, a morphism $f_{1}$ from $a$ to $b$, and a morphism $f_{2}$ from $b$ to $c$. Now we state the propositions: 
(30) If $f_{1}$ is epimorphic and $f_{2}$ is epimorphic, then $f_{2} \cdot f_{1}$ is epimorphic. The theorem is a consequence of (22) and (23).

(31) If $f_{2} \cdot f_{1}$ is epimorphic and $\operatorname{hom}(a, b) \neq \emptyset$ and $\operatorname{hom}(b, c) \neq \emptyset$, then $f_{2}$ is epimorphic. The theorem is a consequence of (23).

(32) Let us consider a category $\mathscr{C}$, objects $a, b$ of $\mathscr{C}$, and a morphism $f$ from $a$ to $b$. If $f$ is a retraction, then $f$ is epimorphic. The theorem is a consequence of (23) and (18).

Let $\mathscr{C}$ be a composable category structure with identities, $a, b$ be objects of $\mathscr{C}$, and $f$ be a morphism from $a$ to $b$. We say that $f$ is isomorphism if and only if

(Def. 9) $\operatorname{hom}(a, b) \neq \emptyset$ and $\operatorname{hom}(b, a) \neq \emptyset$ and there exists a morphism $g$ from $b$ to $a$ such that $g \cdot f=\mathrm{id}-a$ and $f \cdot g=\mathrm{id}-b$.

We say that $a$ and $b$ are isomorphic if and only if

(Def. 10) there exists a morphism $f$ from $a$ to $b$ such that $f$ is isomorphism.

Note that $a$ and $b$ are isomorphic if and only if the condition (Def. 11) is satisfied.

(Def. 11) $\operatorname{hom}(a, b) \neq \emptyset$ and $\operatorname{hom}(b, a) \neq \emptyset$ and there exists a morphism $f$ from $a$ to $b$ and there exists a morphism $g$ from $b$ to $a$ such that $g \cdot f=$ id- $a$ and $f \cdot g=$ id- $b$.

Now we state the proposition:

(33) Let us consider a category $\mathscr{C}$, objects $a, b$ of $\mathscr{C}$, and a morphism $f$ from $a$ to $b$. If $f$ is isomorphism, then $f$ is monomorphic and epimorphic. The theorem is a consequence of (28) and (32).

\section{Ordinal Numbers as Categories}

Let $\mathscr{C}$ be a category structure. We say that $\mathscr{C}$ is a preorder if and only if

(Def. 12) for every objects $a, b$ of $\mathscr{C}$ and for every morphisms $f_{1}, f_{2}$ of $\mathscr{C}$ such that $f_{1}, f_{2} \in \operatorname{hom}(a, b)$ holds $f_{1}=f_{2}$.

Observe that every category structure which is empty is also a preorder and there exists a category structure which is strict and preorder and every composable category structure with identities which is a preorder is also associative.

Let $\mathscr{C}$ be category structure with identities. The functor RelOb $\mathscr{C}$ yielding a binary relation on $\mathrm{Ob} \mathscr{C}$ is defined by the term

(Def. 13) $\{\langle a, b\rangle$, where $a, b$ are objects of $\mathscr{C}$ : there exists a morphism $f$ of $\mathscr{C}$ such that $f \in \operatorname{hom}(a, b)\}$.

Let $\mathscr{C}$ be an empty category structure with identities. Let us note that $\operatorname{RelOb} \mathscr{C}$ is empty. 
Now we state the propositions:

(34) Let us consider a composable category structure $\mathscr{C}$ with identities. Then

(i) $\operatorname{dom} \operatorname{RelOb} \mathscr{C}=\mathrm{Ob} \mathscr{C}$, and

(ii) $\operatorname{rng} \operatorname{RelOb} \mathscr{C}=\mathrm{Ob} \mathscr{C}$.

The theorem is a consequence of (6) and (19).

(35) Let us consider composable category structures $\mathscr{C}_{1}, \mathscr{C}_{2}$ with identities. Suppose $\mathscr{C}_{1} \cong \mathscr{C}_{2}$. Then RelOb $\mathscr{C}_{1}$ and RelOb $\mathscr{C}_{2}$ are isomorphic. The theorem is a consequence of (15), (34), and (20).

Let $\mathscr{C}$ be a non empty, composable category structure with identities. One can verify that $\mathrm{RelOb} \mathscr{C}$ is non empty.

Now we state the propositions:

(36) Let us consider preorder, composable category structure $\mathscr{C}$ with identities. Suppose $\mathscr{C}$ is not empty. Then there exists a function $\mathcal{F}$ from $\mathscr{C}$ into $\operatorname{RelOb} \mathscr{C}$ such that

(i) $\mathcal{F}$ is bijective, and

(ii) for every morphism $f$ of $\mathscr{C}, \mathcal{F}(f)=\langle\operatorname{dom} f$, $\operatorname{cod} f\rangle$.

Proof: Reconsider $\mathscr{C}_{1}=\mathscr{C}$ as a non empty, composable category structure with identities. Define $\mathcal{P}$ [object, object] $\equiv$ for every morphism $f$ of $\mathscr{C}_{1}$ such that $\$_{1}=f$ holds $\$_{2}=\langle\operatorname{dom} f, \operatorname{cod} f\rangle$. For every element $x$ of the carrier of $\mathscr{C}_{1}$, there exists an element $y$ of RelOb $\mathscr{C}_{1}$ such that $\mathcal{P}[x, y]$. Consider $\mathcal{F}$ being a function from the carrier of $\mathscr{C}_{1}$ into RelOb $\mathscr{C}_{1}$ such that for every element $x$ of the carrier of $\mathscr{C}_{1}, \mathcal{P}[x, \mathcal{F}(x)]$ from [10, Sch. 3]. For every object $y$ such that $y \in \operatorname{RelOb} \mathscr{C}$ holds $y \in \operatorname{rng} \mathcal{F}$ by (20), [9, (3)]. For every objects $x_{1}, x_{2}$ such that $x_{1}, x_{2} \in \operatorname{dom} \mathcal{F}$ and $\mathcal{F}\left(x_{1}\right)=\mathcal{F}\left(x_{2}\right)$ holds $x_{1}=x_{2}$.

(37) Let us consider an ordinal number $O$. Then there exists a strict, a preorder category $\mathscr{C}$ such that

(i) $\mathrm{Ob} \mathscr{C}=O$, and

(ii) for every objects $o_{1}, o_{2}$ of $\mathscr{C}$ such that $o_{1} \in o_{2} \operatorname{holds} \operatorname{hom}\left(o_{1}, o_{2}\right)=$ $\left\{\left\langle o_{1}, o_{2}\right\rangle\right\}$, and

(iii) $\operatorname{RelOb} \mathscr{C}=\subseteq_{O}$, and

(iv) Mor $\mathscr{C}=O \cup\left\{\left\langle o_{1}, o_{2}\right\rangle\right.$, where $o_{1}, o_{2}$ are elements of $\left.O: o_{1} \in o_{2}\right\}$.

The theorem is a consequence of (6), (20), and (21).

Let $O$ be an ordinal number and $\mathscr{C}$ be a composable category structure with identities. We say that $\mathscr{C}$ is $O$-ordered if and only if

(Def. 14) RelOb $\mathscr{C}$ and $\subseteq_{O}$ are isomorphic. 
Let $O$ be a non empty, ordinal number. Let us observe that every composable category structure with identities which is $O$-ordered is also non empty.

Let $O$ be an ordinal number. Note that there exists a composable category structure with identities which is strict, $O$-ordered, and preorder.

Let $O$ be an empty, ordinal number. Let us observe that every composable category structure with identities which is $O$-ordered is also empty.

Now we state the proposition:

(38) Let us consider ordinal numbers $O_{1}, O_{2}$, a $O_{1}$-ordered, a preorder category $\mathscr{C}_{1}$, and a $O_{2}$-ordered, a preorder category $\mathscr{C}_{2}$. Then $O_{1}=O_{2}$ if and only if $\mathscr{C}_{1} \cong \mathscr{C}_{2}$.

Proof: If $O_{1}=O_{2}$, then $\mathscr{C}_{1} \cong \mathscr{C}_{2}$ by (13), [4, (39), (41)], (36). If $\mathscr{C}_{1} \cong \mathscr{C}_{2}$, then $O_{1}=O_{2}$ by (35), [4, (42), (40)], [5, (10)].

Let $O$ be an ordinal number. The functor $\mathbf{O}$ yielding a strict, $O$-ordered, a preorder category is defined by the term

(Def. 15) the strict, $O$-ordered, a preorder category.

Now we state the proposition:

(39) There exists a morphism $f$ of $\mathbf{2}$ such that

(i) $f$ is not identity, and

(ii) $\mathrm{Ob} \mathbf{2}=\{\operatorname{dom} f, \operatorname{cod} f\}$, and

(iii) $\operatorname{Mor} \mathbf{2}=\{\operatorname{dom} f, \operatorname{cod} f, f\}$, and

(iv) $\operatorname{dom} f, \operatorname{cod} f, f$ are mutually different.

Proof: Consider $\mathscr{C}$ being a strict, a preorder category such that $\mathrm{Ob} \mathscr{C}=2$ and for every objects $o_{1}, o_{2}$ of $\mathscr{C}$ such that $o_{1} \in o_{2} \operatorname{holds} \operatorname{hom}\left(o_{1}, o_{2}\right)=$ $\left\{\left\langle o_{1}, o_{2}\right\rangle\right\}$ and $\operatorname{RelOb} \mathscr{C}=\subseteq_{2}$ and Mor $\mathscr{C}=2 \cup\left\{\left\langle o_{1}, o_{2}\right\rangle\right.$, where $o_{1}, o_{2}$ are elements of $\left.2: o_{1} \in o_{2}\right\} . \mathscr{C} \cong \mathbf{2}$. Consider $\mathcal{F}$ being a functor from $\mathscr{C}$ to $\mathbf{2}$, $\mathcal{G}$ being a functor from $\mathbf{2}$ to $\mathscr{C}$ such that $\mathcal{F}$ is covariant and $\mathcal{G}$ is covariant and $\mathcal{G} \circ \mathcal{F}=\operatorname{id}_{\mathscr{C}}$ and $\mathcal{F} \circ \mathcal{G}=\operatorname{id}_{\mathbf{2}}$. Reconsider $g=\langle 0,1\rangle$ as a morphism of $\mathscr{C} . g$ is not identity by [17, (22)]. Set $f=\mathcal{F}(g) . f$ is not identity by [9, (18)], [17, (34)]. $\overline{\overline{\mathrm{Ob} 2}}=\overline{\overline{2}}$. Consider $x, y$ being objects such that $x \neq y$ and $\mathrm{Ob} \mathbf{2}=\{x, y\} \operatorname{dom} f \neq \operatorname{cod} f$. For every object $x, x \in$ Mor 2 iff $x \in\{\operatorname{dom} f, \operatorname{cod} f, f\}$ by [17, (22)], [9, (18)], [17, (34)], [2, (50), (49)].

Let $\mathscr{C}$ be a non empty category and $f$ be a morphism of $\mathscr{C}$. The functor $\mathcal{M}_{\mathrm{f}}$ yielding a covariant functor from 2 to $\mathscr{C}$ is defined by

(Def. 16) for every morphism $g$ of $\mathbf{2}$ such that $g$ is not identity holds it $(g)=f$.

Now we state the proposition:

(40) Let us consider a non empty category $\mathscr{C}$, and a morphism $f$ of $\mathscr{C}$. Suppose $f$ is identity. Let us consider a morphism $g$ of $\mathbf{2}$. Then $\left(\mathcal{M}_{\mathrm{f}}\right)(g)=f$. The theorem is a consequence of (39) and (6). 


\section{Pullbacks}

Let $\mathscr{C}$ be a category, $c, c_{1}, c_{2}, d$ be objects of $\mathscr{C}$, and $f_{1}$ be a morphism from $c_{1}$ to $c$. Assume $\operatorname{hom}\left(c_{1}, c\right) \neq \emptyset$. Let $f_{2}$ be a morphism from $c_{2}$ to $c$. Assume $\operatorname{hom}\left(c_{2}, c\right) \neq \emptyset$. Let $p_{1}$ be a morphism from $d$ to $c_{1}$. Assume $\operatorname{hom}\left(d, c_{1}\right) \neq$ $\emptyset$. Let $p_{2}$ be a morphism from $d$ to $c_{2}$. Assume $\operatorname{hom}\left(d, c_{2}\right) \neq \emptyset$. We say that $\left\langle d, p_{1}, p_{2}\right\rangle$ is a pullback of $f_{1}, f_{2}$ if and only if

(Def. 17) $f_{1} \cdot p_{1}=f_{2} \cdot p_{2}$ and for every object $d_{1}$ of $\mathscr{C}$ and for every morphism $g_{1}$ from $d_{1}$ to $c_{1}$ and for every morphism $g_{2}$ from $d_{1}$ to $c_{2}$ such that $\operatorname{hom}\left(d_{1}, c_{1}\right) \neq \emptyset$ and $\operatorname{hom}\left(d_{1}, c_{2}\right) \neq \emptyset$ and $f_{1} \cdot g_{1}=f_{2} \cdot g_{2}$ holds hom $\left(d_{1}, d\right) \neq$ $\emptyset$ and there exists a morphism $h$ from $d_{1}$ to $d$ such that $p_{1} \cdot h=g_{1}$ and $p_{2} \cdot h=g_{2}$ and for every morphism $h_{1}$ from $d_{1}$ to $d$ such that $p_{1} \cdot h_{1}=g_{1}$ and $p_{2} \cdot h_{1}=g_{2}$ holds $h=h_{1}$.

Now we state the proposition:

(41) Let us consider a category $\mathscr{C}$, objects $c, c_{1}, c_{2}, d, e$ of $\mathscr{C}$, a morphism $f_{1}$ from $c_{1}$ to $c$, a morphism $f_{2}$ from $c_{2}$ to $c$, a morphism $p_{1}$ from $d$ to $c_{1}$, a morphism $p_{2}$ from $d$ to $c_{2}$, a morphism $q_{1}$ from $e$ to $c_{1}$, and a morphism $q_{2}$ from $e$ to $c_{2}$. Suppose $\operatorname{hom}\left(c_{1}, c\right) \neq \emptyset$ and $\operatorname{hom}\left(c_{2}, c\right) \neq \emptyset$ and $\operatorname{hom}\left(d, c_{1}\right) \neq \emptyset$ and $\operatorname{hom}\left(d, c_{2}\right) \neq \emptyset$ and $\operatorname{hom}\left(e, c_{1}\right) \neq \emptyset$ and $\operatorname{hom}\left(e, c_{2}\right) \neq \emptyset$ and $\left\langle d, p_{1}, p_{2}\right\rangle$ is a pullback of $f_{1}, f_{2}$ and $\left\langle e, q_{1}, q_{2}\right\rangle$ is a pullback of $f_{1}, f_{2}$. Then $d$ and $e$ are isomorphic. The theorem is a consequence of (23) and (18).

Let us consider a category $\mathscr{C}$, objects $c, c_{1}, c_{2}, d$ of $\mathscr{C}$, a morphism $f_{1}$ from $c_{1}$ to $c$, a morphism $f_{2}$ from $c_{2}$ to $c$, a morphism $p_{1}$ from $d$ to $c_{1}$, and a morphism $p_{2}$ from $d$ to $c_{2}$. Now we state the propositions:

(42) Suppose $\operatorname{hom}\left(c_{1}, c\right) \neq \emptyset$ and $\operatorname{hom}\left(c_{2}, c\right) \neq \emptyset$ and $\operatorname{hom}\left(d, c_{1}\right) \neq \emptyset$ and $\operatorname{hom}\left(d, c_{2}\right) \neq \emptyset$ and $\left\langle d, p_{1}, p_{2}\right\rangle$ is a pullback of $f_{1}, f_{2}$.

Then $\left\langle d, p_{2}, p_{1}\right\rangle$ is a pullback of $f_{2}, f_{1}$.

(43) Suppose $\operatorname{hom}\left(c_{1}, c\right) \neq \emptyset$ and $\operatorname{hom}\left(c_{2}, c\right) \neq \emptyset$ and $\operatorname{hom}\left(d, c_{1}\right) \neq \emptyset$ and $\operatorname{hom}\left(d, c_{2}\right) \neq \emptyset$ and $\left\langle d, p_{1}, p_{2}\right\rangle$ is a pullback of $f_{1}, f_{2}$ and $f_{1}$ is monomorphic. Then $p_{2}$ is monomorphic. The theorem is a consequence of (22) and (23).

(44) Suppose $\operatorname{hom}\left(c_{1}, c\right) \neq \emptyset$ and $\operatorname{hom}\left(c_{2}, c\right) \neq \emptyset$ and $\operatorname{hom}\left(d, c_{1}\right) \neq \emptyset$ and $\operatorname{hom}\left(d, c_{2}\right) \neq \emptyset$ and $\left\langle d, p_{1}, p_{2}\right\rangle$ is a pullback of $f_{1}, f_{2}$ and $f_{1}$ is isomorphism. Then $p_{2}$ is isomorphism. The theorem is a consequence of (22), (23), and (18).

(45) Let us consider a category $\mathscr{C}$, objects $c_{1}, c_{1}, c_{2}, c_{3}, c_{4}, c_{5}, c_{6}$ of $\mathscr{C}$, a morphism $f_{1}$ from $c_{1}$ to $c_{2}$, a morphism $f_{2}$ from $c_{2}$ to $c_{3}$, a morphism $f_{3}$ from $c_{1}$ to $c_{4}$, a morphism $f_{4}$ from $c_{2}$ to $c_{5}$, a morphism $f_{5}$ from 
$c_{3}$ to $c_{6}$, a morphism $f_{6}$ from $c_{4}$ to $c_{5}$, and a morphism $f_{7}$ from $c_{5}$ to $c_{6}$. Suppose $\operatorname{hom}\left(c_{1}, c_{2}\right) \neq \emptyset$ and $\operatorname{hom}\left(c_{2}, c_{3}\right) \neq \emptyset$ and $\operatorname{hom}\left(c_{1}, c_{4}\right) \neq$ $\emptyset$ and $\operatorname{hom}\left(c_{2}, c_{5}\right) \neq \emptyset$ and $\operatorname{hom}\left(c_{3}, c_{6}\right) \neq \emptyset$ and $\operatorname{hom}\left(c_{4}, c_{5}\right) \neq \emptyset$ and $\operatorname{hom}\left(c_{5}, c_{6}\right) \neq \emptyset$ and $\left\langle c_{2}, f_{2}, f_{4}\right\rangle$ is a pullback of $f_{5}, f_{7}$. Then $\left\langle c_{1}, f_{1}, f_{3}\right\rangle$ is a pullback of $f_{4}, f_{6}$ if and only if $\left\langle c_{1}, f_{2} \cdot f_{1}, f_{3}\right\rangle$ is a pullback of $f_{5}, f_{7} \cdot f_{6}$ and $f_{4} \cdot f_{1}=f_{6} \cdot f_{3}$. The theorem is a consequence of (22) and (23).

\section{Pullbacks of Functors}

Let $\mathscr{C}, \mathscr{D}$ be categories and $\mathcal{F}$ be a functor from $\mathscr{C}$ to $\mathscr{D}$. We say that $\mathcal{F}$ is monomorphic if and only if

(Def. 18) $\mathcal{F}$ is covariant and for every category $\mathscr{B}$ and for every functors $\mathcal{G}_{1}, \mathcal{G}_{2}$ from $\mathscr{B}$ to $\mathscr{C}$ such that $\mathcal{G}_{1}$ is covariant and $\mathcal{G}_{2}$ is covariant and $\mathcal{F} \circ \mathcal{G}_{1}=$ $\mathcal{F} \circ \mathcal{G}_{2}$ holds $\mathcal{G}_{1}=\mathcal{G}_{2}$.

We say that $\mathcal{F}$ is isomorphism if and only if

(Def. 19) $\mathcal{F}$ is covariant and there exists a functor $\mathcal{G}$ from $\mathscr{D}$ to $\mathscr{C}$ such that $\mathcal{G}$ is covariant and $\mathcal{G} \circ \mathcal{F}=\operatorname{id}_{\mathscr{C}}$ and $\mathcal{F} \circ \mathcal{G}=\mathrm{id}_{\mathscr{D}}$.

Let $\mathscr{C}, \mathscr{C}_{1}, \mathscr{C}_{2}, \mathscr{D}$ be categories and $\mathcal{F}_{1}$ be a functor from $\mathscr{C}_{1}$ to $\mathscr{C}$. Assume $\mathcal{F}_{1}$ is covariant. Let $\mathcal{F}_{2}$ be a functor from $\mathscr{C}_{2}$ to $\mathscr{C}$. Assume $\mathcal{F}_{2}$ is covariant. Let $\mathcal{P}_{1}$ be a functor from $\mathscr{D}$ to $\mathscr{C}_{1}$. Assume $\mathcal{P}_{1}$ is covariant. Let $\mathcal{P}_{2}$ be a functor from $\mathscr{D}$ to $\mathscr{C}_{2}$. Assume $\mathcal{P}_{2}$ is covariant. We say that $\left\langle\mathscr{D}, \mathcal{P}_{1}, \mathcal{P}_{2}\right\rangle$ is a pullback of $\mathcal{F}_{1}, \mathcal{F}_{2}$ if and only if

(Def. 20) $\mathcal{F}_{1} \circ \mathcal{P}_{1}=\mathcal{F}_{2} \circ \mathcal{P}_{2}$ and for every category $\mathscr{D}_{1}$ and for every functor $\mathcal{G}_{1}$ from $\mathscr{D}_{1}$ to $\mathscr{C}_{1}$ and for every functor $\mathcal{G}_{2}$ from $\mathscr{D}_{1}$ to $\mathscr{C}_{2}$ such that $\mathcal{G}_{1}$ is covariant and $\mathcal{G}_{2}$ is covariant and $\mathcal{F}_{1} \circ \mathcal{G}_{1}=\mathcal{F}_{2} \circ \mathcal{G}_{2}$ there exists a functor $\mathcal{H}$ from $\mathscr{D}_{1}$ to $\mathscr{D}$ such that $\mathcal{H}$ is covariant and $\mathcal{P}_{1} \circ \mathcal{H}=\mathcal{G}_{1}$ and $\mathcal{P}_{2} \circ \mathcal{H}=\mathcal{G}_{2}$ and for every functor $\mathcal{H}_{1}$ from $\mathscr{D}_{1}$ to $\mathscr{D}$ such that $\mathcal{H}_{1}$ is covariant and $\mathcal{P}_{1} \circ \mathcal{H}_{1}=\mathcal{G}_{1}$ and $\mathcal{P}_{2} \circ \mathcal{H}_{1}=\mathcal{G}_{2}$ holds $\mathcal{H}=\mathcal{H}_{1}$.

Now we state the proposition:

(46) Let us consider categories $\mathscr{C}, \mathscr{C}_{1}, \mathscr{C}_{2}, \mathscr{D}, \mathscr{E}$, a functor $\mathcal{F}_{1}$ from $\mathscr{C}_{1}$ to $\mathscr{C}$, a functor $\mathcal{F}_{2}$ from $\mathscr{C}_{2}$ to $\mathscr{C}$, a functor $\mathcal{P}_{1}$ from $\mathscr{D}$ to $\mathscr{C}_{1}$, a functor $\mathcal{P}_{2}$ from $\mathscr{D}$ to $\mathscr{C}_{2}$, a functor $\mathcal{Q}_{1}$ from $\mathscr{E}$ to $\mathscr{C}_{1}$, and a functor $\mathcal{Q}_{2}$ from $\mathscr{E}$ to $\mathscr{C}_{2}$. Suppose $\mathcal{F}_{1}$ is covariant and $\mathcal{F}_{2}$ is covariant and $\mathcal{P}_{1}$ is covariant and $\mathcal{P}_{2}$ is covariant and $\mathcal{Q}_{1}$ is covariant and $\mathcal{Q}_{2}$ is covariant and $\left\langle\mathscr{D}, \mathcal{P}_{1}, \mathcal{P}_{2}\right\rangle$ is a pullback of $\mathcal{F}_{1}, \mathcal{F}_{2}$ and $\left\langle\mathscr{E}, \mathcal{Q}_{1}, \mathcal{Q}_{2}\right\rangle$ is a pullback of $\mathcal{F}_{1}, \mathcal{F}_{2}$. Then $\mathscr{D} \cong \mathscr{E}$.

ProOF: There exists a functor $\mathcal{F}_{8}$ from $\mathscr{D}$ to $\mathscr{E}$ and there exists a functor $\mathcal{G}_{3}$ from $\mathscr{E}$ to $\mathscr{D}$ such that $\mathcal{F}_{8}$ is covariant and $\mathcal{G}_{3}$ is covariant and $\mathcal{G}_{3} \circ \mathcal{F}_{8}=\mathrm{id} \mathscr{D}$ and $\mathcal{F}_{8} \circ \mathcal{G}_{3}=\mathrm{id}_{\mathscr{E}}$ by (10), (11), [17, (35)]. 
Let us consider categories $\mathscr{C}, \mathscr{C}_{1}, \mathscr{C}_{2}, \mathscr{D}$, a functor $\mathcal{F}_{1}$ from $\mathscr{C}_{1}$ to $\mathscr{C}$, a functor $\mathcal{F}_{2}$ from $\mathscr{C}_{2}$ to $\mathscr{C}$, a functor $\mathcal{P}_{1}$ from $\mathscr{D}$ to $\mathscr{C}_{1}$, and a functor $\mathcal{P}_{2}$ from $\mathscr{D}$ to $\mathscr{C}_{2}$. Now we state the propositions:

(47) Suppose $\mathcal{F}_{1}$ is covariant and $\mathcal{F}_{2}$ is covariant and $\mathcal{P}_{1}$ is covariant and $\mathcal{P}_{2}$ is covariant and $\left\langle\mathscr{D}, \mathcal{P}_{1}, \mathcal{P}_{2}\right\rangle$ is a pullback of $\mathcal{F}_{1}, \mathcal{F}_{2}$.

Then $\left\langle\mathscr{D}, \mathcal{P}_{2}, \mathcal{P}_{1}\right\rangle$ is a pullback of $\mathcal{F}_{2}, \mathcal{F}_{1}$.

(48) Suppose $\mathcal{F}_{1}$ is covariant and $\mathcal{F}_{2}$ is covariant and $\mathcal{P}_{1}$ is covariant and $\mathcal{P}_{2}$ is covariant and $\left\langle\mathscr{D}, \mathcal{P}_{1}, \mathcal{P}_{2}\right\rangle$ is a pullback of $\mathcal{F}_{1}, \mathcal{F}_{2}$ and $\mathcal{F}_{1}$ is monomorphic. Then $\mathcal{P}_{2}$ is monomorphic.

PROOF: For every category $\mathscr{D}_{1}$ and for every functors $\mathcal{Q}_{1}, \mathcal{Q}_{2}$ from $\mathscr{D}_{1}$ to $\mathscr{D}$ such that $\mathcal{Q}_{1}$ is covariant and $\mathcal{Q}_{2}$ is covariant and $\mathcal{P}_{2} \circ \mathcal{Q}_{1}=\mathcal{P}_{2} \circ \mathcal{Q}_{2}$ holds $\mathcal{Q}_{1}=\mathcal{Q}_{2}$ by [17, (35)], (10).

(49) Suppose $\mathcal{F}_{1}$ is covariant and $\mathcal{F}_{2}$ is covariant and $\mathcal{P}_{1}$ is covariant and $\mathcal{P}_{2}$ is covariant and $\left\langle\mathscr{D}, \mathcal{P}_{1}, \mathcal{P}_{2}\right\rangle$ is a pullback of $\mathcal{F}_{1}, \mathcal{F}_{2}$ and $\mathcal{F}_{1}$ is isomorphism. Then $\mathcal{P}_{2}$ is isomorphism. The theorem is a consequence of (10) and (11).

(50) Let us consider categories $\mathscr{C}_{1}, \mathscr{C}_{2}, \mathscr{C}_{3}, \mathscr{C}_{4}, \mathscr{C}_{5}, \mathscr{C}_{6}$, a functor $\mathcal{F}_{1}$ from $\mathscr{C}_{1}$ to $\mathscr{C}_{2}$, a functor $\mathcal{F}_{2}$ from $\mathscr{C}_{2}$ to $\mathscr{C}_{3}$, a functor $\mathcal{F}_{3}$ from $\mathscr{C}_{1}$ to $\mathscr{C}_{4}$, a functor $\mathcal{F}_{4}$ from $\mathscr{C}_{2}$ to $\mathscr{C}_{5}$, a functor $\mathcal{F}_{5}$ from $\mathscr{C}_{3}$ to $\mathscr{C}_{6}$, a functor $\mathcal{F}_{6}$ from $\mathscr{C}_{4}$ to $\mathscr{C}_{5}$, and a functor $\mathcal{F}_{7}$ from $\mathscr{C}_{5}$ to $\mathscr{C}_{6}$. Suppose $\mathcal{F}_{1}$ is covariant and $\mathcal{F}_{2}$ is covariant and $\mathcal{F}_{3}$ is covariant and $\mathcal{F}_{4}$ is covariant and $\mathcal{F}_{5}$ is covariant and $\mathcal{F}_{6}$ is covariant and $\mathcal{F}_{7}$ is covariant and $\left\langle\mathscr{C}_{2}, \mathcal{F}_{2}, \mathcal{F}_{4}\right\rangle$ is a pullback of $\mathcal{F}_{5}, \mathcal{F}_{7}$. Then $\left\langle\mathscr{C}_{1}, \mathcal{F}_{1}, \mathcal{F}_{3}\right\rangle$ is a pullback of $\mathcal{F}_{4}, \mathcal{F}_{6}$ if and only if $\left\langle\mathscr{C}_{1}, \mathcal{F}_{2} \circ \mathcal{F}_{1}, \mathcal{F}_{3}\right\rangle$ is a pullback of $\mathcal{F}_{5}, \mathcal{F}_{7} \circ \mathcal{F}_{6}$ and $\mathcal{F}_{4} \circ \mathcal{F}_{1}=\mathcal{F}_{6} \circ \mathcal{F}_{3}$.

PROOF: For every category $\mathscr{D}_{1}$ and for every functor $\mathcal{G}_{1}$ from $\mathscr{D}_{1}$ to $\mathscr{C}_{2}$ and for every functor $\mathcal{G}_{2}$ from $\mathscr{D}_{1}$ to $\mathscr{C}_{4}$ such that $\mathcal{G}_{1}$ is covariant and $\mathcal{G}_{2}$ is covariant and $\mathcal{F}_{4} \circ \mathcal{G}_{1}=\mathcal{F}_{6} \circ \mathcal{G}_{2}$ there exists a functor $\mathcal{H}$ from $\mathscr{D}_{1}$ to $\mathscr{C}_{1}$ such that $\mathcal{H}$ is covariant and $\mathcal{F}_{1} \circ \mathcal{H}=\mathcal{G}_{1}$ and $\mathcal{F}_{3} \circ \mathcal{H}=\mathcal{G}_{2}$ and for every functor $\mathcal{H}_{1}$ from $\mathscr{D}_{1}$ to $\mathscr{C}_{1}$ such that $\mathcal{H}_{1}$ is covariant and $\mathcal{F}_{1} \circ \mathcal{H}_{1}=\mathcal{G}_{1}$ and $\mathcal{F}_{3} \circ \mathcal{H}_{1}=\mathcal{G}_{2}$ holds $\mathcal{H}=\mathcal{H}_{1}$ by [17, (35)], (10).

(51) Let us consider categories $\mathscr{C}, \mathscr{C}_{1}, \mathscr{C}_{2}$, a functor $\mathcal{F}_{1}$ from $\mathscr{C}_{1}$ to $\mathscr{C}$, and a functor $\mathcal{F}_{2}$ from $\mathscr{C}_{2}$ to $\mathscr{C}$. Suppose $\mathcal{F}_{1}$ is covariant and $\mathcal{F}_{2}$ is covariant. Then there exists a strict category $\mathscr{D}$ and there exists a functor $\mathcal{P}_{1}$ from $\mathscr{D}$ to $\mathscr{C}_{1}$ and there exists a functor $\mathcal{P}_{2}$ from $\mathscr{D}$ to $\mathscr{C}_{2}$ such that the carrier of $\mathscr{D}=\left\{\left\langle f_{1}, f_{2}\right\rangle\right.$, where $f_{1}$ is a morphism of $\mathscr{C}_{1}, f_{2}$ is a morphism of $\mathscr{C}_{2}: f_{1} \in$ the carrier of $\mathscr{C}_{1}$ and $f_{2} \in$ the carrier of $\mathscr{C}_{2}$ and $\left.\mathcal{F}_{1}\left(f_{1}\right)=\mathcal{F}_{2}\left(f_{2}\right)\right\}$ and the composition of $\mathscr{D}=\left\{\left\langle\left\langle f_{1}, f_{2}\right\rangle, f_{3}\right\rangle\right.$, where $f_{1}, f_{2}, f_{3}$ are morphisms of $\mathscr{D}: f_{1}, f_{2}, f_{3} \in$ the carrier of $\mathscr{D}$ and for every morphisms $f_{11}, f_{12}, f_{13}$ of $\mathscr{C}_{1}$ and for every morphisms $f_{21}, f_{22}, f_{23}$ of $\mathscr{C}_{2}$ such that $f_{1}=\left\langle f_{11}, f_{21}\right\rangle$ and $f_{2}=\left\langle f_{12}, f_{22}\right\rangle$ and $f_{3}=\left\langle f_{13}, f_{23}\right\rangle$ holds 
$f_{11} \triangleright f_{12}$ and $f_{21} \triangleright f_{22}$ and $f_{13}=f_{11} \circ f_{12}$ and $\left.f_{23}=f_{21} \circ f_{22}\right\}$ and $\mathcal{P}_{1}$ is covariant and $\mathcal{P}_{2}$ is covariant and $\left\langle\mathscr{D}, \mathcal{P}_{1}, \mathcal{P}_{2}\right\rangle$ is a pullback of $\mathcal{F}_{1}, \mathcal{F}_{2}$.

Proof: Reconsider $c_{7}=\left\{\left\langle f_{1}, f_{2}\right\rangle\right.$, where $f_{1}$ is a morphism of $\mathscr{C}_{1}, f_{2}$ is a morphism of $\mathscr{C}_{2}: f_{1} \in$ the carrier of $\mathscr{C}_{1}$ and $f_{2} \in$ the carrier of $\mathscr{C}_{2}$ and $\left.\mathcal{F}_{1}\left(f_{1}\right)=\mathcal{F}_{2}\left(f_{2}\right)\right\}$ as a set. Set $c_{8}=\left\{\left\langle\left\langle x_{1}, x_{2}\right\rangle, x_{3}\right\rangle\right.$, where $x_{1}, x_{2}, x_{3}$ are elements of $c_{7}: x_{1}, x_{2}, x_{3} \in c_{7}$ and for every morphisms $f_{11}, f_{12}, f_{13}$ of $\mathscr{C}_{1}$ and for every morphisms $f_{21}, f_{22}, f_{23}$ of $\mathscr{C}_{2}$ such that $x_{1}=\left\langle f_{11}, f_{21}\right\rangle$ and $x_{2}=\left\langle f_{12}, f_{22}\right\rangle$ and $x_{3}=\left\langle f_{13}, f_{23}\right\rangle$ holds $f_{11} \triangleright f_{12}$ and $f_{21} \triangleright f_{22}$ and $f_{13}=f_{11} \circ f_{12}$ and $\left.f_{23}=f_{21} \circ f_{22}\right\}$. For every object $x$ such that $x \in c_{8}$ holds $x \in\left(c_{7} \times c_{7}\right) \times c_{7}$. For every objects $x, y_{1}, y_{2}$ such that $\left\langle x, y_{1}\right\rangle$, $\left\langle x, y_{2}\right\rangle \in c_{8}$ holds $y_{1}=y_{2}$. Set $\mathscr{D}=\left\langle c_{7}, c_{8}\right\rangle$. For every morphisms $g_{1}$, $g_{2}$ of $\mathscr{D}$ such that $g_{1} \triangleright g_{2}$ there exist morphisms $f_{11}, f_{12}, f_{13}$ of $\mathscr{C}_{1}$ and there exist morphisms $f_{21}, f_{22}, f_{23}$ of $\mathscr{C}_{2}$ such that $g_{1}=\left\langle f_{11}, f_{21}\right\rangle$ and $g_{2}=\left\langle f_{12}, f_{22}\right\rangle$ and $\mathcal{F}_{1}\left(f_{11}\right)=\mathcal{F}_{2}\left(f_{21}\right)$ and $\mathcal{F}_{1}\left(f_{12}\right)=\mathcal{F}_{2}\left(f_{22}\right)$ and $f_{11} \triangleright f_{12}$ and $f_{21} \triangleright f_{22}$ and $f_{13}=f_{11} \circ f_{12}$ and $f_{23}=f_{21} \circ f_{22}$ and $g_{1} \circ g_{2}=\left\langle f_{13}, f_{23}\right\rangle$ by (1), [17, (1)], [9, (1)]. For every morphisms $g_{1}, g_{2}$ of $\mathscr{D}$ such that there exist morphisms $f_{11}, f_{12}$ of $\mathscr{C}_{1}$ and there exist morphisms $f_{21}, f_{22}$ of $\mathscr{C}_{2}$ such that $g_{1}=\left\langle f_{11}, f_{21}\right\rangle$ and $g_{2}=\left\langle f_{12}, f_{22}\right\rangle$ and $\mathcal{F}_{1}\left(f_{11}\right)=\mathcal{F}_{2}\left(f_{21}\right)$ and $\mathcal{F}_{1}\left(f_{12}\right)=\mathcal{F}_{2}\left(f_{22}\right)$ and $f_{11} \triangleright f_{12}$ and $f_{21} \triangleright f_{22}$ holds $g_{1} \triangleright g_{2}$ by (1), [17, (1)]. For every morphisms $g, g_{1}, g_{2}$ of $\mathscr{D}$ such that $g_{1} \triangleright g_{2}$ holds $g_{1} \circ g_{2} \triangleright g$ iff $g_{2} \triangleright g$. For every morphisms $g, g_{1}, g_{2}$ of $\mathscr{D}$ such that $g_{1} \triangleright g_{2}$ holds $g \triangleright g_{1} \circ g_{2}$ iff $g \triangleright g_{1}$. For every morphism $g_{1}$ of $\mathscr{D}$ such that $g_{1} \in$ the carrier of $\mathscr{D}$ there exists a morphism $g$ of $\mathscr{D}$ such that $g \triangleright g_{1}$ and $g$ is left identity by (2), [17. (31), (32)]. For every morphism $g_{1}$ of $\mathscr{D}$ such that $g_{1} \in$ the carrier of $\mathscr{D}$ there exists a morphism $g$ of $\mathscr{D}$ such that $g_{1} \triangleright g$ and $g$ is right identity by (2), [17, (31), (32)]. For every morphisms $g_{1}, g_{2}, g_{3}$ of $\mathscr{D}$ such that $g_{1} \triangleright g_{2}$ and $g_{2} \triangleright g_{3}$ and $g_{1} \circ g_{2} \triangleright g_{3}$ and $g_{1} \triangleright g_{2} \circ g_{3}$ holds $g_{1} \circ\left(g_{2} \circ g_{3}\right)=$ $\left(g_{1} \circ g_{2}\right) \circ g_{3}$. For every object $x, x \in c_{8}$ iff $x \in\left\{\left\langle\left\langle f_{1}, f_{2}\right\rangle, f_{3}\right\rangle\right.$, where $f_{1}, f_{2}, f_{3}$ are morphisms of $\mathscr{D}: f_{1}, f_{2}, f_{3} \in$ the carrier of $\mathscr{D}$ and for every morphisms $f_{11}, f_{12}, f_{13}$ of $\mathscr{C}_{1}$ and for every morphisms $f_{21}, f_{22}, f_{23}$ of $\mathscr{C}_{2}$ such that $f_{1}=\left\langle f_{11}, f_{21}\right\rangle$ and $f_{2}=\left\langle f_{12}, f_{22}\right\rangle$ and $f_{3}=\left\langle f_{13}, f_{23}\right\rangle$ holds $f_{11} \triangleright f_{12}$ and $f_{21} \triangleright f_{22}$ and $f_{13}=f_{11} \circ f_{12}$ and $\left.f_{23}=f_{21} \circ f_{22}\right\}$. There exists a functor $\mathcal{P}_{1}$ from $\mathscr{D}$ to $\mathscr{C}_{1}$ and there exists a functor $\mathcal{P}_{2}$ from $\mathscr{D}$ to $\mathscr{C}_{2}$ such that $\mathcal{P}_{1}$ is covariant and $\mathcal{P}_{2}$ is covariant and $\mathcal{F}_{1} \circ \mathcal{P}_{1}=\mathcal{F}_{2} \circ \mathcal{P}_{2}$ and for every category $\mathscr{D}_{1}$ and for every functor $\mathcal{G}_{1}$ from $\mathscr{D}_{1}$ to $\mathscr{C}_{1}$ and for every functor $\mathcal{G}_{2}$ from $\mathscr{D}_{1}$ to $\mathscr{C}_{2}$ such that $\mathcal{G}_{1}$ is covariant and $\mathcal{G}_{2}$ is covariant and $\mathcal{F}_{1} \circ \mathcal{G}_{1}=\mathcal{F}_{2} \circ \mathcal{G}_{2}$ there exists a functor $\mathcal{H}$ from $\mathscr{D}_{1}$ to $\mathscr{D}$ such that $\mathcal{H}$ is covariant and $\mathcal{P}_{1} \circ \mathcal{H}=\mathcal{G}_{1}$ and $\mathcal{P}_{2} \circ \mathcal{H}=\mathcal{G}_{2}$ and for every functor $\mathcal{H}_{1}$ from $\mathscr{D}_{1}$ to $\mathscr{D}$ such that $\mathcal{H}_{1}$ is covariant and $\mathcal{P}_{1} \circ \mathcal{H}_{1}=\mathcal{G}_{1}$ and $\mathcal{P}_{2} \circ \mathcal{H}_{1}=\mathcal{G}_{2}$ holds $\mathcal{H}=\mathcal{H}_{1}$ by [17, (31)], [9, (13)], (1), [17, (32), (34)]. Consider $\mathcal{P}_{1}$ 
being a functor from $\mathscr{D}$ to $\mathscr{C}_{1}, \mathcal{P}_{2}$ being a functor from $\mathscr{D}$ to $\mathscr{C}_{2}$ such that $\mathcal{P}_{1}$ is covariant and $\mathcal{P}_{2}$ is covariant and $\mathcal{F}_{1} \circ \mathcal{P}_{1}=\mathcal{F}_{2} \circ \mathcal{P}_{2}$ and for every category $\mathscr{D}_{1}$ and for every functor $\mathcal{G}_{1}$ from $\mathscr{D}_{1}$ to $\mathscr{C}_{1}$ and for every functor $\mathcal{G}_{2}$ from $\mathscr{D}_{1}$ to $\mathscr{C}_{2}$ such that $\mathcal{G}_{1}$ is covariant and $\mathcal{G}_{2}$ is covariant and $\mathcal{F}_{1} \circ \mathcal{G}_{1}=\mathcal{F}_{2} \circ \mathcal{G}_{2}$ there exists a functor $\mathcal{H}$ from $\mathscr{D}_{1}$ to $\mathscr{D}$ such that $\mathcal{H}$ is covariant and $\mathcal{P}_{1} \circ \mathcal{H}=\mathcal{G}_{1}$ and $\mathcal{P}_{2} \circ \mathcal{H}=\mathcal{G}_{2}$ and for every functor $\mathcal{H}_{1}$ from $\mathscr{D}_{1}$ to $\mathscr{D}$ such that $\mathcal{H}_{1}$ is covariant and $\mathcal{P}_{1} \circ \mathcal{H}_{1}=\mathcal{G}_{1}$ and $\mathcal{P}_{2} \circ \mathcal{H}_{1}=\mathcal{G}_{2}$ holds $\mathcal{H}=\mathcal{H}_{1}$.

Let $\mathscr{C}, \mathscr{C}_{1}, \mathscr{C}_{2}$ be categories and $\mathcal{F}_{1}$ be a functor from $\mathscr{C}_{1}$ to $\mathscr{C}$. Assume $\mathcal{F}_{1}$ is covariant. Let $\mathcal{F}_{2}$ be a functor from $\mathscr{C}_{2}$ to $\mathscr{C}$. Assume $\mathcal{F}_{2}$ is covariant.

A pullback of $\mathcal{F}_{1}, \mathcal{F}_{2}$ is a triple object and is defined by

(Def. 21) there exists a strict category $\mathscr{D}$ and there exists a functor $\mathcal{P}_{1}$ from $\mathscr{D}$ to $\mathscr{C}_{1}$ and there exists a functor $\mathcal{P}_{2}$ from $\mathscr{D}$ to $\mathscr{C}_{2}$ such that it $=\left\langle\mathscr{D}, \mathcal{P}_{1}, \mathcal{P}_{2}\right\rangle$ and $\mathcal{P}_{1}$ is covariant and $\mathcal{P}_{2}$ is covariant and $\left\langle\mathscr{D}, \mathcal{P}_{1}, \mathcal{P}_{2}\right\rangle$ is a pullback of $\mathcal{F}_{1}, \mathcal{F}_{2}$.

Assume $\mathcal{F}_{1}$ is covariant. Assume $\mathcal{F}_{2}$ is covariant. The functor $\llbracket \mathcal{F}_{1}, \mathcal{F}_{2} \rrbracket$ yielding a strict category is defined by the term

(Def. 22) the pullback of $\mathcal{F}_{1}, \mathcal{F}_{21,3}$.

Assume $\mathcal{F}_{1}$ is covariant. Assume $\mathcal{F}_{2}$ is covariant. The functor $\pi_{1}\left(\mathcal{F}_{1} \otimes \mathcal{F}_{2}\right)$ yielding a functor from $\llbracket \mathcal{F}_{1}, \mathcal{F}_{2} \rrbracket$ to $\mathscr{C}_{1}$ is defined by the term

(Def. 23) the pullback of $\mathcal{F}_{1}, \mathcal{F}_{22,3}$.

The functor $\pi_{2}\left(\mathcal{F}_{1} \otimes \mathcal{F}_{2}\right)$ yielding a functor from $\llbracket \mathcal{F}_{1}, \mathcal{F}_{2} \rrbracket$ to $\mathscr{C}_{2}$ is defined by the term

(Def. 24) the pullback of $\mathcal{F}_{1}, \mathcal{F}_{23,3}$.

Let us consider categories $\mathscr{C}, \mathscr{C}_{1}, \mathscr{C}_{2}$, a functor $\mathcal{F}_{1}$ from $\mathscr{C}_{1}$ to $\mathscr{C}$, and a functor $\mathcal{F}_{2}$ from $\mathscr{C}_{2}$ to $\mathscr{C}$. Let us assume that $\mathcal{F}_{1}$ is covariant and $\mathcal{F}_{2}$ is covariant. Now we state the propositions:

(i) $\pi_{1}\left(\mathcal{F}_{1} \otimes \mathcal{F}_{2}\right)$ is covariant, and

(ii) $\pi_{2}\left(\mathcal{F}_{1} \otimes \mathcal{F}_{2}\right)$ is covariant, and

(iii) $\left\langle\llbracket \mathcal{F}_{1}, \mathcal{F}_{2} \rrbracket, \pi_{1}\left(\mathcal{F}_{1} \otimes \mathcal{F}_{2}\right), \pi_{2}\left(\mathcal{F}_{1} \otimes \mathcal{F}_{2}\right)\right\rangle$ is a pullback of $\mathcal{F}_{1}, \mathcal{F}_{2}$.

(53) $\llbracket \mathcal{F}_{1}, \mathcal{F}_{2} \rrbracket \cong \llbracket \mathcal{F}_{2}, \mathcal{F}_{1} \rrbracket$. The theorem is a consequence of (52), (47), and (46).

(54) There exist object-categories $\mathscr{C}, \mathscr{C}_{1}, \mathscr{C}_{2}$ and there exists a functor $\mathcal{F}_{1}$ from $\mathscr{C}_{1}$ to $\mathscr{C}$ and there exists a functor $\mathcal{F}_{2}$ from $\mathscr{C}_{2}$ to $\mathscr{C}$ such that there exists no object-category $\mathscr{D}$ and there exists a functor $\mathcal{P}_{1}$ from $\mathscr{D}$ to $\mathscr{C}_{1}$ and there exists a functor $\mathcal{P}_{2}$ from $\mathscr{D}$ to $\mathscr{C}_{2}$ such that $\mathcal{F}_{1} \cdot \mathcal{P}_{1}=\mathcal{F}_{2} \cdot \mathcal{P}_{2}$ and for every object-category $\mathscr{D}_{1}$ and for every functor $\mathcal{G}_{1}$ from $\mathscr{D}_{1}$ to $\mathscr{C}_{1}$ and for every functor $\mathcal{G}_{2}$ from $\mathscr{D}_{1}$ to $\mathscr{C}_{2}$ such that $\mathcal{F}_{1} \cdot \mathcal{G}_{1}=\mathcal{F}_{2} \cdot \mathcal{G}_{2}$ there exists a functor $\mathcal{H}$ from $\mathscr{D}_{1}$ to $\mathscr{D}$ such that $\mathcal{P}_{1} \cdot \mathcal{H}=\mathcal{G}_{1}$ and $\mathcal{P}_{2} \cdot \mathcal{H}=\mathcal{G}_{2}$ and for 
every functor $\mathcal{H}_{1}$ from $\mathscr{D}_{1}$ to $\mathscr{D}$ such that $\mathcal{P}_{1} \cdot \mathcal{H}_{1}=\mathcal{G}_{1}$ and $\mathcal{P}_{2} \cdot \mathcal{H}_{1}=\mathcal{G}_{2}$ holds $\mathcal{H}=\mathcal{H}_{1}$. The theorem is a consequence of (39) and (40).

\section{REFERENCES}

[1] Jiri Adamek, Horst Herrlich, and George E. Strecker. Abstract and Concrete Categories: The Joy of Cats. Dover Publication, New York, 2009.

[2] Grzegorz Bancerek. Cardinal numbers Formalized Mathematics, 1(2):377-382, 1990.

[3] Grzegorz Bancerek. The ordinal numbers, Formalized Mathematics, 1(1):91-96, 1990.

[4] Grzegorz Bancerek. The well ordering relations. Formalized Mathematics, 1(1):123-129, 1990.

[5] Grzegorz Bancerek. Zermelo theorem and axiom of choice. Formalized Mathematics, 1 (2):265-267, 1990.

[6] Grzegorz Bancerek and Krzysztof Hryniewiecki. Segments of natural numbers and finite sequences. Formalized Mathematics, 1(1):107-114, 1990.

[7] Francis Borceaux. Handbook of Categorical Algebra I. Basic Category Theory, volume 50 of Encyclopedia of Mathematics and its Applications. Cambridge University Press, Cambridge, 1994.

[8] Czesław Byliński. Introduction to categories and functors Formalized Mathematics, 1 (2):409-420, 1990.

[9] Czesław Byliński. Functions and their basic properties Formalized Mathematics, 1(1): 55-65, 1990.

[10] Czesław Byliński. Functions from a set to a set Formalized Mathematics, 1(1):153-164, 1990.

[11] Czesław Byliński. Partial functions. Formalized Mathematics, 1(2):357-367, 1990.

[12] Czesław Byliński. Some basic properties of sets Formalized Mathematics, 1(1):47-53, 1990.

[13] Agata Darmochwał. Finite sets. Formalized Mathematics, 1(1):165-167, 1990.

[14] F. William Lawvere. Functorial semantics of algebraic theories and some algebraic problems in the context of functorial semantics of algebraic theories. Reprints in Theory and Applications of Categories, 5:1-121, 2004.

[15] Saunders Mac Lane. Categories for the Working Mathematician, volume 5 of Graduate Texts in Mathematics. Springer Verlag, New York, Heidelberg, Berlin, 1971.

[16] Beata Padlewska. Families of sets Formalized Mathematics, 1(1):147-152, 1990.

[17] Marco Riccardi. Obiect-free definition of categories. Formalized Mathematics, 21(3): 193-205, 2013. doi $10.2478 /$ forma-2013-0021

[18] Andrzej Trybulec. Enumerated sets. Formalized Mathematics, 1(1):25-34, 1990.

[19] Zinaida Trybulec. Properties of subsets Formalized Mathematics, 1(1):67-71, 1990.

[20] Edmund Woronowicz. Relations and their basic properties. Formalized Mathematics, 1 (1):73-83, 1990.

[21] Edmund Woronowicz. Relations defined on sets. Formalized Mathematics, 1(1):181-186, 1990. 